\title{
Research on Service Driven Based Management Information System Reengineering in Cloud Environment
}

\author{
Yang Xiang ${ }^{1, a}$, Shengbin Jia ${ }^{2, b}$, Bo Zhang ${ }^{3}$, Dongdong LV ${ }^{1}$, \\ Dong Wang ${ }^{4}$, Li Yang ${ }^{5}$ \\ ${ }^{1}$ College of Electronic and Information Engineering, Tongji University, Shanghai, 201804, China \\ ${ }^{2}$ College of Information Science and Engineer, Shandong University of Science and Technology, \\ Qingdao, 266510, China \\ ${ }^{3}$ College of Information, Mechanical and Electrical Engineering, Shanghai Normal University, \\ Shanghai, 201804, China \\ ${ }^{4}$ Department of Computer Science and Information Engineering, Shanghai Institute of Technology, \\ Shanghai, 201804, China \\ ${ }^{5}$ Changzhou city people's procuratorate, Changzhou, 213000, China \\ aemail: shxiangyang@tongji.edu.cn. bemail:luckyinfinite@163.com
}

Keywords: management information, system reengineering, cloud computing, information service, semantic normal form.

\begin{abstract}
With the emergence of cloud era, varieties of data resources, computing resources as well as service resources have been greatly enriched by many existing cloud platforms. Although these cloud resources have brought new opportunities for management information system reengineering, we are still facing the challenge of the contradictions about "service standardization and personalized demand" or "quality of service and cost of reengineering". In particular, for this challenge, this research project is intended to build the semantic normal form for management information system reengineering based on semantic computation in cloud environments. As a result, the built semantic normal form can be used to implement the standardized descriptions of user requirement semantics, information resource semantics, information service semantics and service process semantics, as well as the transformation calculation. On the basis above, we propose a set of semantic-based methods for requirement understanding, resources calculation, service computing and process reconstruction. Finally, we form a kind of new theory and methodology for service-driven based management information system reengineering in cloud environments, which will be provided to explore an innovation way for the development of management information systems.
\end{abstract}

\section{Introduction}

In the environment of fierce competition and changing market demand, business processes is difficult to solidify. If the process changed, the companies would have to face enormous challenges [1]. Thus traditional management information systems reengineering faces the contradictions about "service standardization and personalized demand" or "quality of service and cost of reengineering", which are difficult to reconcile. However, the arrival of the era of cloud, for the management of information systems reengineering opens a window.

Cloud environment for the management information system reengineering to provide the most reliable and most secure network data storage center, elastically scalable hardware and software that reduces system maintenance costs and easily shares data between different devices, on-demand billing charges mode [2]. Through invoking, combining or calculating service on the cloud, we can reengineer management information systems to meet customer needs, so that satisfy the individual requirement of the destination user. Therefore, to change the traditional management information systems reengineering model, using the service-driven based management information system reengineering in cloud environment is an effective way. 
However, the cloud also brings many challenges to management information system reengineering. Mainly reflects in: (1) Extremely enriched service stimulates personalized requirement in cloud environment, the requirement is difficult to be accurately analyzed; (2) There are so much service and service resource in cloud environment, how can we effectively integrate various service and service resource in order to reduce the cost of development? (3) Business process reengineering based on cloud service is lack of effective methods.

Therefore, exploring and solving the challenges where are from management information system reengineering in cloud environment, is the important significance of theory and practice. Predictably, with the aid of cloud computing technology of management information system reengineering in cloud environment is the development direction of information system in the future.

\section{Methodology of Proposed Work}

We present the innovative method that service driven based management information system reengineering in cloud environment for the challenge referred to above. In this paper, the program expanded from three levels: (1) Sketch the basic theory of service driven based management information systems reengineering in cloud environment, laying the foundation for semantic normal form; (2) Define a new paradigm of semantic system to lays the foundation for resolutions of the four puzzles what are requirement understanding, resources calculation, service computing and process reconstruction; (3) Explore above four puzzle solutions.

Semantic Computing for System reengineering in Cloud Environment. "Semantics" is the meaning of the concepts that represent the real world things that data corresponds to, and is the relationship between these meanings. Its interpretation and logical representation of data on a field [3]. Semantic computing is complex analysis operation in order to enable the computer to understand the link between information based on semantic description [4].

The information system in the cloud environment is highly autonomous and intelligent, where the information resource, information service and other objects must have a high understanding and interoperability, which requires all kinds of objects within the system must have a good semantic description, to finish semantic computing better. We use the ontology as a means to service semantic representation [5]. Ontology provides a formal, structured knowledge representation and has the characteristics of reuse and sharing [6]. We represent the service objects, such as user requirement, information resource, information service and service process, by the Characterized Cluster Diagram. And then use a common ontology to build its Service Characteristics Ontology. Which provides both structural description and calculated logical basis for these elements.

Information Service for System reengineering in Cloud Environment. Cloud service needs to complete the calculation focused on the cloud environment. In order to take full advantage of cloud environment's characteristic, such as virtualization, flexible, low cost, high flexibility, we should give service the semantic expression that has the ability to properly structure service, so that we can better accomplish service computing $[7,8]$.

We can give an extended service definition [9], where a service $s$ is a triple: $\left\langle{ }^{\circ} s, s^{\circ}, O\right\rangle$ Wherein, 's represents the sets of input parameters of the service, $s$ indicates the sets of output parameters of service, $O$ represents a collection of other service elements, for example behavioral constraints, service quality and so on.

The key of information service is to meet the needs of users. Corresponding to the formal description of the information service, the needs of users $Q$ can be defined as a tuple: $\left\langle Q_{p}, Q_{r}\right\rangle$ which $Q_{p}$ represents a collection of user-supplied parameters, $Q_{r}$ represents a collection of parameters for user needs. Each step in Service Composition [10] needs a process that, according to the current sets of parameters, lookup service next callable. We call this process as Service Retrieve, whose formal description is as follows:

$$
\operatorname{Re}(A, S)=\{s \mid \cdot s \subseteq A \wedge s \in S\}
$$

Where, $A$ is a parameter sets. $S$ is a set of services. Re $(A, S)$ shows that all input parameters of the 
service which can be found from $S$ are included in $A$. With the above result, Service Discovery can be rewritten as:

$$
D c(Q, L(O), S)=\left\{s \mid \operatorname{Re}\left(Q_{p}, S\right) \wedge Q_{r} \subseteq\left(Q_{p} \cup s^{*}\right) s . O \angle L(O)\right\}
$$

The meaning of this formula is that the operations of Service Discovery can only work from the result of Service Retrieval, instead of having to search in the whole service database. Thus, it greatly reduces the search space of Service Discovery and the time of Service Discovery and Service Composition.

The SNF System for Management Information System Reengineering. Compute will be focus to the clouds. However, these multi-source heterogeneous four elements, including user requirement, information resource, information service and service process, bring understanding, interoperability issues to computer. Therefore, we propose Semantic Normal Form (SNF) system in cloud environment, which characterizes the extent of the semantic description of each element and gets the methods of conversion process between the paradigms, through semantic description specifications of different levels of the paradigm. So that the elements can be exchange to more accurate semantic description by the transformation methods, and the system can perform calculations on the basis of understanding.

In terms of demand, resource, service, process the four key elements, Semantic Normal Form is composed of four specific semantic paradigm:

(1). Requirement Semantic Normal Form (QSHF).

Definition: It's a specification about different levels of understandable semantic description of user requirement, as well as conversion guidelines between different levels of semantic description.

Classification:

1QSNF: Can be determined as the semantic description of the effective requirement (validity).

2QSNF: To meet the requirement of $1 \mathrm{QSNF}$ and can mark characteristic (characterization).

3QSNF: To meet the requirement of 2QSNF and can be marked by ontology (completeness).

Conversion Guidelines: Ontology annotation feature (1QSNF to 2QSNF), ontology algebra, temporal logic (2QSNF to 3QSNF).

(2). Resource Semantic Normal Form (RSNF).

Definition: It refers to a specification about different levels of computable semantic description of cloud resource, as well as conversion guidelines between different levels of semantic description.

Classification:

1RSNF: Can be identified and determined to the semantic description of resource (decidability).

2RSNF: To meet the requirement of $1 \mathrm{RSNF}$ and can mark characteristic of knowledge (tagging).

3RSNF: To meet 2RSNF and to determine the usability (availability).

Conversion Guidelines: The annotating conversion of ontology knowledge (1RSNF to 2RSNF), ontology algebra, temporal logic (2RSNF to 3RSNF).

(3). Service Semantic Normal Form (SSNF).

Definition: It's a specification about different levels of computable semantic description of cloud service, as well as conversion guidelines between different levels of semantic description.

Classification:

1SSNF: Service semantic description can be discovered (existence).

2SSNF: To meet the 1SSNF and can determine the composition (adaptation).

3SSNF: To meet the 2SSNF and can be verified (correctness).

Conversion Guidelines: Ontology semantic annotation and semantic computation (1SSNF to 2SSNF), formal reasoning and ontology algebra transformation (2SSNF to 3SSNF).

(4). Flow Semantic Normal Form (FSNF).

Definition: It's a specification about different levels of semantic description from standardized to personalize of business processes, as well as conversion guidelines between different levels of semantic description.

Classification:

1FSNF: basic business process, the link is not variable (standardized process).

2FSNF: to meet the 1FSNF and can be reconstructed (flexible process). 
3FSNF: to meet the 2FSNF and can realize requirement (personalized process).

Conversion Guidelines: Ontology knowledge, temporal logic and 2SNF (1FSNF to 2FSNF), temporal logic, process algebra, and 3SSNF (2FSNF to 3FSNF).

On the basis of four semantic paradigms and its transformation rules, on the integration of description logic [7], temporal logic, ontology algebra and other formal verification methods, we verify the correctness and completeness of the semantic paradigms and its transformation process.

\section{Semantic Normal Form based System reengineering in Cloud Environment}

The Research on User Requirement Understanding Based on QSNF. User requirement is the most important driving force of implementing information service on the cloud platform. We achieve the Requirement Semantic by conversion from effectiveness judgment, characteristic labeling to ontology semantic description layer by layer, in order to achieve requirement understand. In 1QSNF level, the user requirement is described in an unstructured manner, with understandable foundation and structuring possibility. During the conversion from 1QSNF to 2QSNF, the characteristic, field and process of specific user requirement are tagged semi-structurally, given the key features of requirement. The transformation from $3 \mathrm{QSNF}$ to $2 \mathrm{QSNF}$, achieves unique description, proving verification, demand collaboration and process optimization to user requirement semantic, response to time sequence and uncertainty of information service requirement process.

The Research of Information Resource Integration Based on RSNF. We give the description of Resource Semantic based on RSNF, so that these resource have a standardized description, which has greater shared degree and reusability. 1RSNF to 2RSNF semantic transformation extracts key features to tag from the non-structural semantic description, so that the computer can rely on the 2RSNF, which is semi structured semantic level, to identify the resource. The semantic conversion from 2RSNF to 3RSNF, will accurately describe the non-structural description of 2RSNF in terms of the methods of fine-grained annotation and relational reasoning, which makes the computer to uniquely understand resource in the semantic description of 3RSNF. Secondly, after obtaining the standard description of resource, we research the resource self-discovery method, which can achieve classifying resource to different situations according to the resource description, in order to make the system fully understand the resource semantic. Further, the resource adaptation theory is given to realize the resource integration on the basis of semantic standardization.

The Research of Information Service Computing Based on SSNF. Information service in the cloud environment must ensure its availability, adaptation and correctness, to meet the requirement of large-scale, autonomous computing. Therefore, it is necessary for research of information service computing to under the foundation of semantic description which must can show the above three characteristics.

Firstly, we propose the different levels of service semantic description methods and transformation rules basing on the SSNF. In 1SSNF, the system can accurately identify the semantic of a service in the function, location, call interface, etc. in order to understand the availability of the service. From 1SSNF to 2SSNF, we give explicit rules for the serving adaptation; Service achieves the judgment of crossing platform seamless combination based on 2SSNF. The conversion from 2SSNF to 3SSNF, gives explicit rules of enforceable service, and the execution semantics of service which can ensure that service under 3SSNF is verifiable in terms of prediction, reasoning. Secondly, based on the above described methods and transformation rules of SSNF semantics, we offer the service discovery, composition, verification methods. Finally, in order to guarantee the logical tight coupling, which guarantees the availability of service, between service and resource, we should set up a mapping between the resource semantic and service semantic through semantic correlation measure.

The Research of Service Flow Reengineering Based on FSNF. The standard of service flow avoids the inconsistency of the functional expression of the service flow, so that the service can be interconnected and combined normatively. But the standardized flow can't meet the customer's personalized requirement. It never rains but it pours, the flow customization for the personalized requirement will bring a huge cost pressure.

The flow design is divided into three stages, which is "the standardized process $\rightarrow$ the 
reconfigurable process $\rightarrow$ the personalized process". In the first stage, we give the 1FSNF semantic description to assume a service must go through the "standardized process". In the second stage, we use the methods of transforming from 1FSNF to 2FSNF to complete the flow reengineering, including the logical operations such as the link merging, the link replacement, and the sequence reconstruction and so on. In 2FSNF, the flow semantics is divided into the determination link and the reconstruction link. The third stage, we transform the reconstruction link of the 2FSNF to determinate link which meets the needs of users, and depict the only flow according to the process algebra, the time sequence logic, etc. In 3FSNF, the system can perform a service task by understanding and implementing the flow semantics directly.

\section{Conclusion}

Based on the innovation of management information system reengineering in cloud environment. We build the semantic normal form under the foundation of semantic computation in cloud environments. On the basis above, we form a kind of new methodology for service-driven based management information system reengineering in cloud environments, which effectively alleviates the contradictions about "service standardization and personalized demand" or "quality of service and cost of reengineering". Of course, due to time and other reasons, the theoretical model has many details are not perfect, there are shortcomings.

\section{Acknowledgement}

In this paper, the research was sponsored by the National Natural Science Foundation of China (Project No. 71571136), and the National Basic Research Program of China (Project No. 2014CB340404).

\section{References}

[1] Yaqin Sun, Wei Tang, Urban geological environment information system based on three tier C/S structure, J, Computer Engineering, China, 2006.

[2] Seiji Ysunobu, Kuniaki Matsumoto. Information service system, Tokyo, 1991.

[3] Qin Chunxiu, Research Review on Semantics Analysis of Natural Language, Library and Information Service, Vol. 58, Issue 22, 2014.

[4] Salton, Gerard, Buckley, C. Term-weighting approaches in automatic text retrieval. Information Processing \& Management. 24 (1988) 513-523.

[5] Shouli Tang, Research on Ontology-based Cloud Services Semantic Retrieval System, New Technology of Library and Information Service, Issue 12, 2014.

[6] Fensel D. Ontologies: A Silver Bullet for Knowledge Management and Electronic Commerce. Springer. (2003) 90-96.

[7] Zhiyong Fen, Service Network Prototype System, Computer Science, Vol. 41, Issue 9, 2014.

[8] Chuang Lin, A survey on models and solutions of multi-objective optimization for QoS in services computing, Chinese Journal of Computers, Vol. 38, Issue 10, 2015.

[9] Jinhua Li, Semantic query, matching and combination of service discovery oriented scientific workflow, Information Studies: Theory \& Application, Vol. 38 Issue 6, 2015.

[10] Weifeng Pan, Service composition recommendation based on service networks, Systems Engineering- Theory \& Practice, Vol. 34, Suppl, 2014. 